\title{
Summer reading: popular science and 'lab lit'
}

An idiosyncratic selection of science-themed books for the summer.

Warm weather is upon us here at the Nature Methods editorial office, and we hope our readers have at least some chance to stretch out in the sunshine with a cold drink and an engrossing book. Should one want to stay close to matters scientific, there are plenty of sciencethemed books to enjoy.

Popular scientific nonfiction is typically intended for a general audience, but these stories can also be fascinating to scientists who want to broaden their horizons. Some works in this genre can help refine the public perception of a scientific field. Two noteworthy examples tackling the topic of evolution include The Accidental Species: Misunderstandings of Human Evolution by Henry Gee and Undeniable: Evolution and the Science of Creation by Bill Nye. The former, written by a paleontologist and evolutionary biologist (who is also an editor at Nature), presents the study of evolution in clear terms, discussing what fossil records can and cannot tell researchers and explaining why some popular concepts, such as 'the missing link', are fallacious. The latter, by the famous TV personality Bill Nye (the Science Guy), takes on the topic of evolution with the wit for which he is beloved, bringing the theory to life even for those who find evolution undeniable in the first place.

For those who want to view their own work through a wider lens, Microcosm: E. coli and the New Science of Life by the popular science writer Carl Zimmer is sure to please. This book takes readers on a journey through the history of biologists' most widely used model organism. After thinking about the roles of E. coli-from our labs to our guts-and the parallels between their genome and ours, the flasks of bacteria shaking in the incubator may never look the same.

What If?: Serious Scientific Answers to Absurd Hypothetical Questions by Randall Munroe is a great choice for anyone who has ever wondered whether it is possible to build a jetpack using downward-firing machine guns, or what would happen if all the water in a storm fell as one giant raindrop. Munroe, a former NASA roboticist and founder of the popular webcomic $x k c d$, presents well-researched answers to such bizarre questions in a way that ensures both a smile and relief that rain falls in droplets.

As an alternative to popular science, a fiction genre called 'lab lit' has also grown steadily in recent years, with some titles even appearing as New York Times best sellers, such as State of Wonder and Flight Behavior, discussed below, as well as others such as Orfeo by Richard Powers and Euphoria by Lily King. In lab lit, scientists in realistic settings are either the protagonists of the story or are central to the plot. Such novels often emphasize that scientists, like other people, face ethical and personal dilemmas both in and out of the laboratory.

In State of Wonder by Ann Patchett, a scientific researcher at a pharmaceutical company must travel deep into the Amazon to check up on her secretive former mentor who may have discovered the cure for infertility. The novel can be read as a twist on Joseph Conrad's Heart of Darkness and, like that novella, takes the protagonist deep into the mysteries that wait in the jungle and sends her home much changed.

Generosity: An Enhancement by Richard Powers centers on what might happen in a world with a happiness gene. In the novel, a woman with "an excess of happiness" agrees to be studied by a geneticist who discovers the genetic basis for happiness. The novel highlights the implications of understanding our genetic identities, such as genome engineering to make designer humans, while maintaining the humanity of the characters. Powers is well known for bringing scientific concepts into literature, and this book is an excellent example of his work.

Another recent example of lab lit takes on the topical issue of climate change. In Flight Behavior by Barbara Kingsolver, the protagonist discovers that monarch butterflies have migrated to her family's property in rural Appalachia-property that she and her family had planned to $\log$ for money to save their farm-rather than to Mexico for the winter. The presence of the butterflies is seen by some as a miracle and by others as a dire sign of climate change, and it draws an entomologist and his laboratory into their midst to study the butterflies' strange behavior. The novel takes on science, faith, the economy and life decisions with honesty and compassion, and it features a scientist who is at times out of his element in rural Tennessee.

These titles are just a sampling from a number of exemplary books with scientific themes from past several years. Our selection is not intended to be complete or authoritative, but is influenced by our own reading and is meant as a starting point. We hope our readers enjoy them as much as we did. 